\title{
Association of Low Umbilical Cord pH and Hypoglycemia in Healthy Term Newborns: Should it Be a Part of Postnatal Screening?
}

\author{
Naveed Ur Rehman Durrani ${ }^{1}$, Jameel AlGhamdi², Niels Rochow1, Salhab el Helou1 and Michael Lefebvre Marrin1 \\ ${ }^{1}$ Department of Pediatrics, Neonatal Division. McMaster Children Hospital, Hamilton, Canada \\ ${ }^{2}$ Department of Pediatrics, Al-Baha University, Saudi Arabia
}

\begin{abstract}
Hypoglycemia may lead to neurological impairment; therefore, high-risk newborns are screened postnatally. However, hypoglycemia monitoring protocols often do not include cord blood acidosis as a risk factor. The study aimed to find an association between asymptomatic cord blood acidosis and hypoglycemia. All healthy term infants born at McMaster Children Hospital, Hamilton, Canada, between October 2013 and September 2014, who had umbilical cord blood pH $<7.0$ or base excess $\leq 12 \mathrm{mmol} / \mathrm{L}$ were studied. Infants with evidence of hypoxic-ischemic encephalopathy, birth weights outside of $10^{\text {th }}$ to $95^{\text {th }}$ percentiles for gestation, mothers with preeclampsia, diabetes or taking a $\beta$-blockers at the time of the birth were excluded. Hypoglycemia was defined as blood glucose $<2.6 \mathrm{mmol} / \mathrm{L}$ in the first two hours of life. One hundred sixtysix infants met the cord blood gas criteria, but only 16 had hypoglycemia. Although infants with mild perinatal depression are at risk for hypoglycemia, a significant association could not be proved.
\end{abstract}

Key Words: Umbilical cord pH, Hypoglycemia, Neonate, Term, Screening, Acidosis.

How to cite this article: Durrani NUR, AIGhamdi J, Rochow N, Helou SE, Marrin ML. Association of low umbilical cord pH and hypoglycemia in healthy term newborns: should it be a part of postnatal screening? J Coll Physicians Surg Pak 2019; 29(11):1116-7.

Glucose is the primary energy substrate for the newborn brain.1,2 Transient hypoglycemia during the first few hours of life is a common phenomenon. 3,4 Some infants who have experienced intrauterine hypoxia-ischemia have transient hypoglycemia. Hypothesized mechanisms include the inefficiency of anaerobic ATP synthesis and hyperinsulinism. 2,5

Although intrauterine hypoxia-ischemia is a potential risk factor for transient hypoglycemia, our nursery hypoglycemia surveillance protocol did not include infants who had cord blood acidosis but appeared neurologically well. The authors were unable to identify any studies on the risk of hypoglycemia in such infants. After identifying hypoglycemia in two infants whose only potential perinatal risk factor was an asymptomatic cord blood acidosis, the authors undertook this retrospective study to explore a potential association.

This was a single-centre, retrospective study of infants born between October 2013 to September 2014, who were admitted to the Children's Hospital Level II or III nurseries for hypoglycemia without apparent risk factors for hypoglycemia apart from an umbilical cord $\mathrm{pH}$ of $<7.0$ or base excess $\leq 12 \mathrm{mmol} / \mathrm{L}$.

Correspondence to: Dr. Naveed Ur Rehman Durrani,

Department of NICU, McMaster Children Hospital, 1280

Main Street West, Hamilton, ON L8S4k1, Canada

E-mail: ndurrani@npmmcmaster.ca

Received: October 15, 2018; Revised: April 01, 2019;

Accepted: April 12, 2019
Data were abstracted from the electronic medical records in two phases. In phase 1, the cord blood gases of all term infants admitted to Level II or III nurseries for management of hypoglycemia were reviewed. In phase 2, following the implementation of a cord acidosis as a potential risk factor, blood glucose concentrations of all apparently healthy term newborn infants with a cord arterial or venous $\mathrm{pH}<7.0$ or base excess $\leq 12 \mathrm{mmol} / \mathrm{L}$ were recorded. These healthy newborns were defined as having a gestational age $\geq 37$ weeks and $<42$ weeks of gestational age who did not require admission to special care nursery.

The dataset included maternal history, gestational age, and known risk factors for hypoglycemia. Small for gestational age was defined as a birth weight $<10^{\text {th }}$ percentile for the gestational age. Large for gestational age was defined as birth weight $>90^{\text {th }}$ percentile for gestational age. Also excluded were infants with cultureproven sepsis, infants born to mothers with diabetes (whether gestational or type I or type 2) and infants born to mothers receiving beta-blockers or other medications known to be associated with newborn hypoglycemia. Hypoglycemia was defined as blood glucose <2.6 $\mathrm{mmol} / \mathrm{L}$. Acidosis and hypoglycemia were analysed using a $2 \times 2$ crosstabulation. Also, odds ratios, positive predictive value, negative predictive value and $95 \%$ confidence intervals were calculated. To investigate the relation between acidosis and glucose levels, linear and logistic regression models, as well as Chi-square test and Fisher's exact test, were performed. The level of 
Table I: Characteristics of infants with acidosis and hypoglycemia.

\begin{tabular}{|c|c|c|c|c|c|c|}
\hline Location & GA (weeks) & Sex & Weight (gm) & ABG ph/BE (mmol/L) & VBG $\mathrm{ph} / \mathrm{BE}(\mathrm{mmol} / \mathrm{L})$ & Age at first glucose \\
\hline $\mathrm{L} 2 \mathrm{~N}$ & $40+6 / 7$ & Female & 3925 & $6.91 /-16$ & $6.95 /-15$ & 1.5 at $6 \mathrm{hrs}$ of age \\
\hline $\mathrm{NICU}$ & 37 & Male & 3130 & $6.96 /-16$ & $7.02 /-15$ & 2.1 at $3 \mathrm{hrs}$ of age \\
\hline L2N & $38+5 / 7$ & Female & 2552 & $7.11 /-10$ & $6.92 /-16$ & 0.8 at $3 \mathrm{hrs}$ of age \\
\hline L2N & $40+1 / 7$ & Male & 3870 & $7.02 /-12$ & $7.10 /-12$ & 0.4 at $2 \mathrm{hrs}$ of age \\
\hline $\mathrm{NICU}$ & 41 & Female & 3160 & $7.02 /-11$ & $7.20 /-11$ & 2.0 at $2 \mathrm{hrs}$ of age \\
\hline $\mathrm{NICU}$ & 37 & Male & 2170 & $7.03 /-13$ & $7.03 /-14$ & 2.4 at $5 \mathrm{hrs}$ of age \\
\hline
\end{tabular}

L2N: Level II nursery, GA: Gestational age, ABG: Arterial blood gas, BE: Base excess, VBG: Venous blood gas.

Table II: Association between acidosis, hypoglycemia and p-value.

\begin{tabular}{l|c|c|c}
\hline & $\begin{array}{c}\text { Hypoglycemia } \\
<2.6 \mathrm{mmol} / \mathrm{L}\end{array}$ & $\begin{array}{c}\text { No Hypoglycemia } \\
\geq 2.6 \mathrm{mmol} / \mathrm{L}\end{array}$ & p-value \\
\hline $\begin{array}{l}\text { Acidosis } \\
\mathrm{pH}<7.0 \text { or base excess }\end{array}$ & $16(36.4 \%)$ & $28(63.6 \%)$ & \multirow{2}{*}{0.863} \\
$\leq 12 \mathrm{mmol} / \mathrm{L}$ & $3(33.3 \%)$ & $6(66.7 \%)$ & \\
\hline $\begin{array}{l}\text { No acidosis } \\
\mathrm{pH} \geq 7.0 \text { or base excess }\end{array}$ & & & \\
$\leq 12 \mathrm{mmol} / \mathrm{L}$ & &
\end{tabular}

Table III: Odd ratio, positive predictive value and negative predictive value with $95 \%$ confidence interval.

\begin{tabular}{l|c|c}
\hline Quantities derived from 2x2 table & Value & 95\% Confidence interval \\
\hline Odd ratio & 1.143 & $(0.251-5.204)$ \\
Positive predictive value & 0.364 & $(0.222-0.506)$ \\
Negative predictive value & 0.667 & $(0.359-0.975)$ \\
\hline
\end{tabular}

significance was set to $p<0.05$. The statistical analysis was conducted using SPSS version 22, Microsoft Excel ${ }^{\circledR}$ version 2016 and $R$ software package for statistical analysis, R Foundation for Statistical Computing, version 3.5.0 (2018-04-23).

During phase 1, 161 newborns were admitted to the neonatal nurseries with hypoglycemia (blood glucose $<2.6 \mathrm{mmol} / \mathrm{L})$. Of these, 142 had normal cord gases. Of the 19 infants who had a cord blood acidosis, 13 had known risk factors for hypoglycemia (five insulin dependent diabetes mother, two gestational diabetes, one LGA, one SGA, one mother on labetalol, one asphyxia, one sepsis). The six infants who had asymptomatic cord blood acidosis as the only potential risk factor required intravenous dextrose for 2-4 days. Their length of stay was 4-9 days. The characteristics of these six infants are shown in Table I.

On the speculation, the asymptomatic infants with a cord blood acidosis were at risk for hypoglycemia, this factor was included in a revised glucose screening guideline. Thus we were able to assess this association. Of 2,423 infants, admitted to the maternal-newborn unit, 166 $(6.9 \%)$ term infants met the cord gas criteria for acidosis. Despite the new guideline, only $53(31.9 \%)$ had blood glucose measured in the first hour of life. Of 44 babies with cord gases showing acidosis, 16 (36.4\%) had hypoglycemia; and 9 babies with no acidosis, 3 (33.3\%) had hypoglycemia. Several regression models were tested accounting for umbilical arterial and venous $\mathrm{pH}$ and base excess, blood glucose level and its timing. The data did not show significant relations $(p=0.863)$.
In addition, cross tabulation for base excess of $\leq-12$ or $\mathrm{pH}<7.0$ and glucose $<2.6 \mathrm{mmol} / \mathrm{L}$ as cut off for acidosis and hypoglycemia, respectively, revealed a Chi-square value of 0.03 and $p$-value of 0.863 .

There is evidence about the relationship between perinatal distress leading to acidosis and neonatal hypoglycemia. ${ }^{5}$ This study reported on infants who had a cord acidosis but were asymptomatic and developed hypoglycemia in the first 2 days of life. Although, the current retrospective study was unable to show a statistically significant association between asymptomatic cord blood acidosis and transitional hypoglycemia, however we recommend that such babies should continue to be monitored on postnatal ward for hypoglycemia till larger prospective studies confirm or refute the association of cord blood acidosis and blood glucose level of apparently healthy newborns.

\section{ETHICAL APPROVAL:}

Ethical approval from Hamilton Integrated Research Ethics Board was obtained prior to initiation of the sutdy.

\section{CONFLICT OF INTEREST:}

Authors declared no conflict of interest.

\section{AUTHORS' CONTRIBUTION}

NURD: Authored manuscript, and corresponding author with all replies from Journal answering person, literature review. JA: Data collection, literature review.

NR: Data analysis and manuscript proofreading.

$\mathrm{SH}, \mathrm{MM}$ : Reading manuscript for language and grammar correction, proofreading, critical analysis of statistical tests.

\section{REFERENCES}

1. Jones MD, Burd LI, Makowski EI, Meschia G, Battaglia. Cerebral metabolism in sheep: A comparative study in the adult, the lamb and the fetus. Am J Physiol 1975; 229:235-9.

2. Volpe JJ. Neurology of the Newborn. $4^{\text {th }}$ ed. Philadelphia, PA: WB Saunders; 2000:497-520.

3. Stanley CA, Baker L. The causes of neonatal hypoglycemia. N Engl J Med 1999; 18:127-9.

4. Flores-le Roux JA, Sagarra E, Benaiges D, Hernandez-Rivas E, Chillaron JJ, Jaume Puig de Dou, et al. A prospective evaluation of neonatal hypoglycemia in infants of women with gestational diabetes mellitus. Diabetes Res Clin Pract 2012; 97:217-22.

5. Collins JE, Leonard JV. Hyperinsulinism in asphyxiated and small-for-dates infants with hypoglycemia. Lancet 1984; 2:311-3.

$$
\text { …ㄴ..... }
$$

